\title{
Alternative access: jack of all trades or master of one?
}

\author{
Irbaz Hameed ${ }^{1}$, Arash Salemi ${ }^{2}$ \\ ${ }^{1}$ Department of Cardiothoracic Surgery, Weill Cornell Medicine, New York, NY, USA; ${ }^{2}$ Department of Cardiothoracic Surgery, RWJ/Barnabas \\ Health, West Orange, NJ, USA \\ Correspondence to: Arash Salemi, MD, FACS. Department of Cardiothoracic Surgery, RWJ/Barnabas Health, West Orange, NJ, USA. \\ Email: Arash.Salemi@rwjbh.org.
}

Submitted Mar 03, 2020. Accepted for publication May 11, 2020.

doi: $10.21037 /$ acs-2020-av-35

View this article at: http://dx.doi.org/10.21037/acs-2020-av-35

The indications for transcatheter aortic valve replacement (TAVR) have expanded to include patients with symptomatic aortic stenosis at all levels of risk. Over the same timeframe, as TAVR devices and delivery systems have gone through iterative changes, the transfemoral (TF) sheath size has been reduced from 24-Fr down to a much more manageable 14-Fr for many valve sizes. With this evolution, the percentage of alternate access cases has predictably gone down. This reduction has been so substantial that in current practice, with current devices, no more than $5-10 \%$ of cases require a non-TF approach. As such, whereas 5-10 years ago alternative approaches were commonplace, they now are rarely performed. Additionally, with the widespread growth in TAVR programs during the current era of smaller sheaths, many programs lack experience in alternate access. When faced with prohibitive ileo-femoral vasculature, many centers, large and small, are left to choose from a handful of alternative approaches for which local experience is lacking or sparse at best.

The proportion of patients undergoing TAVR by transthoracic alternate access has decreased over the last decade, while the use of peripheral access has remained relatively constant (1). In Europe, data from the French Aortic National CoreValve and Edwards 2 (FRANCE 2) and FRANCE transcatheter aortic valve implantation registries show that alternate access use decreased by $>50 \%$ over the last decade (1). In North America, the Society of Thoracic Surgeons/American College of Cardiology/ Transcatheter Valve Therapy (STS/ACC/TVT) registry shows that the use of alternate access decreased from $24.1 \%$ to $13.4 \%$ between 2012 and 2015 (2).

The choice of one alternate approach over the other remains controversial. Furthermore, evidence on alternate access techniques is based on observational series and no randomized trial has compared their clinical outcomes. The transapical (TA) approach was the first alternative to TF access. However, due to the accumulating evidence on the inferiority of the TA access $v s$. other alternate access techniques and the need for surgical cut-down, general anesthesia and intubation, the use of TA access has progressively decreased among TAVR operators.

More recently, the transaortic (TAo), trans-subclavian (TS), transcarotid (TC), and transcaval (TCv) alternate approaches have emerged. Data from large prospective registries suggest that the TAo access risks higher longterm mortality and adverse event rates than the TF access. Compared to the TA access, however, the TAo approach may improve long-term mortality. The TS access has similar short- and long-term mortality compared to the TF approach, although the incidence of adverse events tends to be higher for TS access. Compared to the TA and TAo accesses, the TS access has better short-term mortality and similar long-term mortality and adverse event rates. However, pacemaker implantation and stroke rates may be higher for the TS compared to the TA and TAo approaches.

The TC and TF approaches demonstrate similar outcomes. Even though a major concern for TC access is an increased risk of stroke, the TC access has been shown to have comparable stroke rates to other alternate accesses. Compared to the transthoracic alternate approaches, TC access is associated with lower incidence of vascular complications. The TC and TS approaches are associated with similar mortality. In the few studies that have reported outcomes for TCv access, short- and long-term mortality as well as adverse event rates are higher than those described 
for other alternative approaches in large registries (1). The $\mathrm{TCv}$ approach is still experimental and should only be performed in patients with favorable abdominal vasculature who are ineligible for TF and other alternate peripheral accesses.

The association between institutional and operator TF TAVR volumes and improved clinical outcomes is well known $(3,4)$. However, data on the volume-outcome relationships or the learning curves for performing TAVR using alternate access is limited and there are no studies on the learning curve for TS and TC approaches.

A recent analysis of 8,644 non-TF TAVR procedures showed that high-volume institutions had significantly lower adjusted 30-day mortality rates than low-volume institutions (3). More specifically, in an analysis of 1,100 patients undergoing TA-TAVR in the Placement of Aortic Transcatheter Valves trial, the learning curve was 30-45 performed procedures. Another study found a statistically significant reduction in the occurrence of major complications after the first 150 TA cases (5). For TAo TAVR, the learning curve for the occurrence of adverse events has been found to be at 128 cases (6). By comparison, the learning curve for TAo TAVR has been determined to be only 20 procedures (7). For TCv TAVR, survival at high-volume centers has been reported to be significantly higher at 30-days but not at 1-year compared to low-volume centers (8).

Alternate access TAVR should be performed by high-volume operators in a hybrid operating room alongside cardiothoracic anesthesiologists and surgeons (9). Additionally, patients should undergo comprehensive diagnostic imaging, including transthoracic echocardiography, multidetector computed tomography, and coronary angiography to assess aortic and vascular anatomy and functionality. Crucially, patient comorbidities, including peripheral artery disease and diabetes, should be considered in conjunction with patient anatomy before performing alternate access TAVR.

TF TAVR remains the primary alternative to surgical aortic valve replacement for intermediate- to prohibitiverisk patients with severe aortic stenosis. The growing popularity of TAVR, however, means that the ability to tailor delivery to patients with unique anatomical challenges using alternate access will become increasingly important to a TAVR operator's practice. While there are no randomized data to support one alternate access over the other, mounting evidence shows a volume-outcome relationship for these approaches. Regionalization of care and experience are important, and institutions and TAVR operators should strive to master one approach. Ultimately, the choice of access must depend on a careful assessment of the individual patient's risk factors and anatomy by the heart team.

\section{Acknowledgments}

Funding: None.

\section{Footnote}

Conflicts of Interest: The authors have no conflicts of interest to declare.

Open Access Statement: This is an Open Access article distributed in accordance with the Creative Commons Attribution-NonCommercial-NoDerivs 4.0 International License (CC BY-NC-ND 4.0), which permits the noncommercial replication and distribution of the article with the strict proviso that no changes or edits are made and the original work is properly cited (including links to both the formal publication through the relevant DOI and the license). See: https://creativecommons.org/licenses/by-nc-nd/4.0/.

\section{References}

1. Beurtheret S, Karam N, Resseguier N, et al. Femoral Versus Nonfemoral Peripheral Access for Transcatheter Aortic Valve Replacement. J Am Coll Cardiol 2019;74:2728-39.

2. Dahle TG, Kaneko T, McCabe JM. Outcomes Following Subclavian and Axillary Artery Access for Transcatheter Aortic Valve Replacement: Society of the Thoracic Surgeons/American College of Cardiology TVT Registry Report. JACC Cardiovasc Interv 2019;12:662-9.

3. Vemulapalli S, Carroll JD, Mack MJ, et al. Procedural Volume and Outcomes for Transcatheter Aortic-Valve Replacement. N Engl J Med 2019;380:2541-50.

4. Salemi A, Sedrakyan A, Mao J, et al. Individual Operator Experience and Outcomes in Transcatheter Aortic Valve Replacement. JACC Cardiovasc Interv 2019;12:90-7.

5. Holzhey DM, Hänsig M, Walther T, et al. Transapical aortic valve implantation - The Leipzig experience. Ann Cardiothorac Surg 2012;1:129-37.

6. Arai T, Romano M, Lefèvre T, et al. Impact of procedural volume on outcome optimization in transaortic transcatheter aortic valve implantation. Int J Cardiol 
2016;223:292-6.

7. Henn MC, Percival T, Zajarias A, et al. Learning Alternative Access Approaches for Transcatheter Aortic Valve Replacement: Implications for New Transcatheter Aortic Valve Replacement Centers. Ann Thorac Surg 2017;103:1399-405.

8. Lederman RJ, Babaliaros VC, Rogers T, et al. The Fate of Transcaval Access Tracts: 12-Month Results of the Prospective NHLBI Transcaval Transcatheter Aortic

Cite this article as: Hameed I, Salemi A. Alternative access: jack of all trades or master of one? Ann Cardiothorac Surg 2020;9(6):496-498. doi: 10.21037/acs-2020-av-35
Valve Replacement Study. JACC Cardiovasc Interv 2019;12:448-56.

9. Otto CM, Kumbhani DJ, Alexander KP, et al. 2017 ACC Expert Consensus Decision Pathway for Transcatheter Aortic Valve Replacement in the Management of Adults With Aortic Stenosis: A Report of the American College of Cardiology Task Force on Clinical Expert Consensus Documents. J Am Coll Cardiol 2017;69:1313-46. 